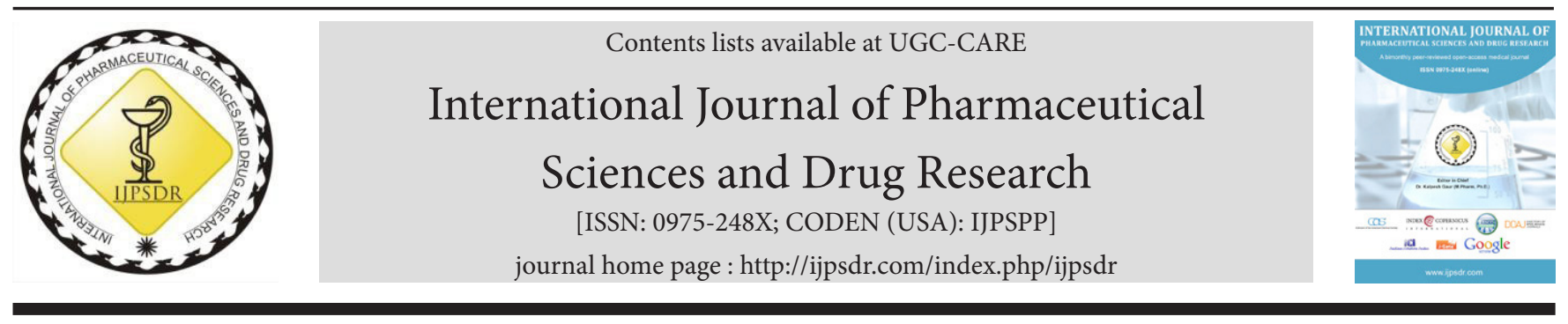

Research Article

\title{
Formulation Evaluation and Optimization of Chitosan Coated Ceftriaxone Loaded Microparticles
}

\author{
Mayuri Jain ${ }^{1}$, Shailja Choudhary ${ }^{1}$, Mojahidul Islam ${ }^{2 *}$ \\ ${ }^{1}$ Department of Pharmaceutics, Amity institute of Pharmacy, Noida-201301, Uttar Pradesh, India \\ ${ }^{2}$ Department of Pharmaceutical Chemistry, School of Pharmacy, Sharda University, Greater Noida-201310, Uttar Pradesh, India
}

\begin{tabular}{l} 
A R T I C L E I N F O \\
\hline Article history: \\
Received: 11 October, 2019 \\
Revised: 03 January, 2020 \\
Accepted: 09 January, 2020 \\
Published: 30 January, 2020 \\
Keywords: \\
Box-Behnken design, \\
Emulsion crosslinking method, \\
Microparticles, \\
MIC. \\
DOI: \\
10.25004/IJPSDR.2020.120105
\end{tabular}

\section{INTRODUCTION}

Ceftriaxone is a third-generation cephalosporin and belongs to biopharmaceutics classification system (BCS) class III and was discovered in 1984 in the United States, and till now the use of the ceftriaxone continuously increases. Ceftriaxone is only available as a parenteral in the market and under the brand name of Rocephin. It can also be given by both intravenous and intramuscular route of administration and is used to treat the various bacterial infection caused by the susceptible microorganisms. Dosage regimen in adults should be $1-2 \mathrm{~g}$, and it is given intramuscularly and intravenously for 7-14 days in one or two divided doses. The parenteral formulation of ceftriaxone was more used medicines for the treatment of serious bacterial infections and it can be effectively administered to the patients with the advanced hepatic syndrome, modification in dose are generally being required mainly for renal failure. Ceftriaxone is only available as a parenteral formulation in the market, so by formulating the microparticles of ceftriaxone drug can be given orally by using microencapsulation technique for the patient compliance and sustained drug release over the long and more period of time. Microparticulate drug delivery dosage form system these systems will provide the sustained and controlled release action of the drug for the more extended period of the time. ${ }^{[1-3]}$ They are freely flowing powder, which consists of proteins and synthetic polymers with the size range from $100-1000 \mathrm{~nm}$. It allows protection of the drug from the environment,

*Corresponding Author: Dr. Mojahidul Islam

Address: Department of Pharmaceutical Chemistry, School of Pharmacy, Sharda University, Greater Noida-201310, Uttar Pradesh, India Email $\bowtie$ : mojahidul.islam@sharda.ac.in

Relevant conflicts of interest/financial disclosures: The authors declare that the research was conducted in the absence of any commercial or financial relationships that could be construed as a potential conflict of interest.

Copyright (c) 2020 Mayuri Jain et al. This is an open access article distributed under the terms of the Creative Commons Attribution- NonCommercialShareAlike 4.0 International License which allows others to remix, tweak, and build upon the work non-commercially, as long as the author is credited and the new creations are licensed under the identical terms. 
in the stabilization of sensitive drugs substance, in the elimination of the incompatibilities, or also in masking the bitter taste. Hence, it has an essential role in increasing the bioavailability of drugs and also reduces the side effects.

\section{MATERIAL AND METHOD}

Ceftriaxone sodium obtained as a sample from Aristo Pharm in Mumbai, Chitosan from Himedia Laboratories Pvt Ltd. Mumbai, Span 80, glacial acetic acid, n-hexane, Glutaraldehyde, Liquid paraffin obtained from Central drug house Pvt. Ltd. New Delhi.

\section{Method Emulsion Crosslinking Method}

Weigh chitosan and ceftriaxone and dissolved in $2 \% \mathrm{v} / \mathrm{v}$ of acetic acid. The drug and polymer mixture was then dissolved in $50 \mathrm{~mL}$ light liquid paraffin containing span $80(0.5 \mathrm{~mL})$ and it was stirred with the help of a magnetic stirrer 1500 revolution per minute (rpm). At an interval of 10 and 40 minutes, glutaraldehyde (GA) was added and stirred continuously till 2 hours. After 2 hours, suspension of chitosan microparticles obtained was then allowed to stand for 15 minutes so that the microparticles will settle down. ${ }^{[4-5]}$ Remaining supernatant was decanted and filtered. Microparticles obtained were then washed four times with n-hexane so that it removes traces of the oil. Then the microparticles were finally washed with water to remove the excess quantity of GA. Dried it at the room temperature for 24 hours and then kept at a temperature of $-80^{\circ} \mathrm{C}$ for 2 hours and then lyophilize it for 24 hours.

\section{Optimization by Box-Behnken Design}

Microparticles formula was optimized by using Box Behnken design expert by taking three independent variables that are polymer concentration, stirring speed, and crosslinking agent concentration and dependent variables are entrapment efficiency and in-vitro release. ${ }^{[6]}$ The present study has a 13 run, 3 factors, 3 level Box Behnken (Table 1) design for the optimization of the microparticles of ceftriaxone employing the Design
Expert Software Stat-Ease (Version 8.0.4, Stat-ease, Inc, Minneapolis, MN). Further studies of optimization were depended on the two dependent variables in-vitro release and entrapment efficiency and the optimized formulation obtained by these two parameters is then evaluated with another evaluation parameter. Fig. 1 shows the variables applied in the design expert software achieved by the preformulation studies of the microparticle

\section{Evaluation of Microparticles}

\section{Particle Size Determination}

- Dynamic light scattering (DLS)

Microparticles sonicated in phosphate buffer at pH 7.4 with volume made up to $10 \mathrm{~mL}$. A total of $1 \mathrm{~mL}$ of this suspension was filled in a cuvette and analyzed for particle size. This was carried out by malvern zeta sizer Ver. 6.00. ${ }^{[7]}$

- Scanning electron microscopy

The determination of particle size and morphology of the surface of the microparticles of ceftriaxone is then carried out by SEM. Samples of SEM was mounted on metal studs and were magnified to X 2000. This was carried out by (ZEISS EVO Special edition. S.NO 65TT/37760/2011/1). ${ }^{[7]}$

\section{Percentage Entrapment Efficiency}

A total of $25 \mathrm{mg}$ of microparticles were crushed and mix the dispersion in $100 \mathrm{~mL}$ of the phosphate buffer of

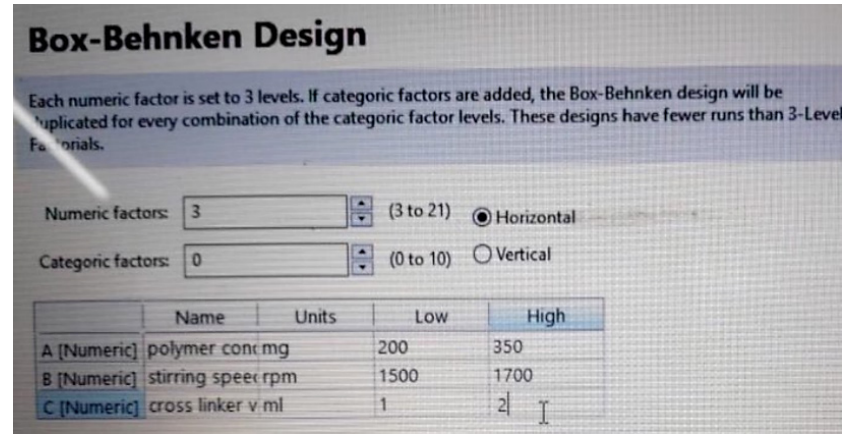

Fig. 1: Optimization technique used for ceftriaxone loaded microparticles

Table 1: Optimization-Box-behnken design

\begin{tabular}{lllll}
\hline \multicolumn{5}{l}{ Formulation table as in the Box-Behnken design } \\
\hline Formulation code & Drug $(\mathrm{mg})$ & Polymer $(\mathrm{mg})$ & Glutaraldehyde $(25 \%)(\mathrm{mL})$ & Stirring speed $(\mathrm{rpm})$ \\
\hline F1 & 50 & 275 & 1 & 1700 \\
F2 & 50 & 350 & 1.5 & 1500 \\
F3 & 50 & 275 & 2 & 1700 \\
F4 & 50 & 350 & 1.5 & 1700 \\
F5 & 50 & 275 & 2 & 1500 \\
F6 & 50 & 350 & 1 & 1600 \\
F7 & 50 & 350 & 1 & 1600 \\
F8 & 50 & 200 & 2 & 1600 \\
F9 & 50 & 275 & 1.5 & 1500 \\
F10 & 50 & 200 & 2 & 1600 \\
F11 & 50 & 275 & 1 & 1600 \\
F12 & 50 & 200 & 1.5 & 1500 \\
F13 & 50 & & & 1700 \\
\hline
\end{tabular}


pH 7.4 and sonicated for 20 minutes. ${ }^{[8-9]}$ Dispersion was stirred in a magnetic stirrer for about 6 hours, and was analyzed spectrophotometrically at $241 \mathrm{~nm}$. Then calculate the percentage drug entrapment efficiency by using the following formula:

\% Entrapment Efficiency : $\frac{\text { Practical drug content }}{\text { Theoretical drug }} \times 100$ content

\section{In-vitro Drug Release}

$20 \mathrm{mg}$ microparticles were taken, which is tied with the dialysis membrane, tie both the ends with a thread. The bag containing formulation was made to hang and rotate over a beaker containing $200 \mathrm{~mL}$ of $\mathrm{pH} 7.4$ phosphate buffer in a way that the bag easily rotates in the buffer by placing the beaker on the magnetic stirrer. The temperature was maintained at $37^{\circ} \mathrm{C}$. At a certain time interval, 5/10 mL of the sample was withdrawn (as per the formulations), which is then replaced with the same amount of buffer freshly made. ${ }^{[10-11]}$ after 24 hours all withdrawn samples were then analyzed by UV spectrophotometer at $241 \mathrm{~nm}$, and absorbance was noted.

\section{In-Vitro Release Kinetics}

Drug release kinetic, done by plotting the graph of different models, Models for kinetics of drug release used are zero-order kinetics, Korsmeyer Peppas model, first-order kinetics, Higuchi Model graph which is fitted with this equation show a straight line. ${ }^{[12-13]}$ The graph, which showed a straight line and good value of regression analysis, was found out to be best.

\section{Minimum Inhibitory Concentration of Ceftriaxone and Ceftriaxone loaded microparticles}

Minimum inhibitory concentration (MIC) studies of ceftriaxone were performed against the strains of bacteria that are Streptococcus mutans, Klebsiella pneumoniae, Staphylococcus aureus, Escherichia coli and Pseudomonas aeruginosa.

MIC studies of the ceftriaxone were studied by using a broth dilution method. MIC studies help establish a resistance to particular bacterial strains. The broth dilution method firstly prepares the stock solution of a different antibiotic concentration of antibiotic stock solution were prepared $(100 \mu \mathrm{g} / \mathrm{mL})$. The inoculum were prepared by using the 18-24 hours agar plate. Adjust the suspension so that it becomes turbid upto the equivalent of $0.5 \mathrm{M}$ cfarland turbidity standard then compare both the tube of inoculum and 0.5 Mcfarland turbidity against the white background after the 15 minutes of the preparation dilute the inoculum suspension in the broth and then add the $1 \mathrm{~mL}$ of the adjusted inoculum suspension to the $1 \mathrm{~mL}$ of the antimicrobial dilution then the inoculation tubes were incubated at $35 \pm 2^{\circ} \mathrm{C}$ for $16-20$ hours in an ambient air incubator then compare both the tubes and wells contain antimicrobial agent with the growing amount of the controlled growth well and the tubes. ${ }^{[14]}$

\section{RESULTS AND DISCUSSION}

\section{Particle Size Determination}

\section{Dynamic Light Scattering}

Determination of Particle size of microparticle was performed by dynamic light scattering and the result of the analysis showed that particle size of microparticles is in the range, and the poly dispersibility index of the microparticle is also less than one, which means formulation F8 was stable. ${ }^{[15]}$ The table given below is the particle size analysis of F8 formulation.

\section{Scanning Electron Microscopy}

The morphology, as well as surface appearance of microparticles, was examined using SEM; the SEM photographs showed that particles have smooth surface. Microparticles formed from this technique were spherical in shape with small size 6-12 $\mu \mathrm{m} .{ }^{[15]}$ SEM images of microparticles are shown in the Fig. 3.

\section{Percentage Entrapment Efficiency}

The entrapment efficiency of microparticle was increase with the increase in the polymer and crosslinking agent and does not show any effect with the increase in stirring speed. F8 formulation has the highest entrapment efficiency because of higher amount of crosslinking agent and higher amount of polymer used in it. F8 formulation entrapment efficiency was found to be $61.7 \%$. The table given below is the entrapment efficiency of all the formulations (Table 3).

\section{In-vitro Drug Release}

In-vitro release of microparticle showed increase in drug release with the increase in time, which shows that drug released in a sustained release manner. The result

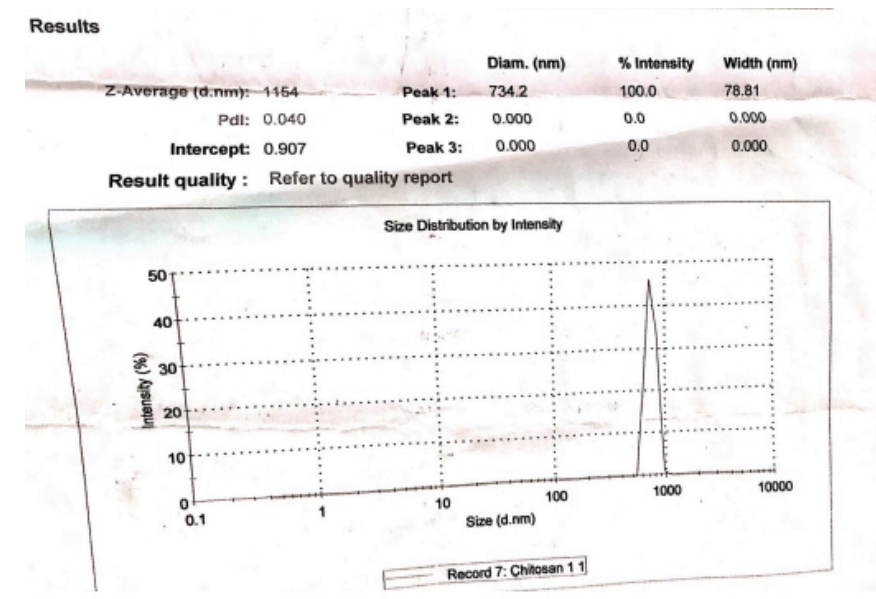

Fig. 2: Dynamic light scattering of ceftriaxone microparticle.

Table 2: Evaluation parameter dynamic light scattering

\begin{tabular}{llll}
\hline S. No & Formulation & Average particle size $(\mathrm{nm})$ & PDI \\
\hline 1 & F8 & 734.2 & 0.040 \\
\hline
\end{tabular}


of the drug release shows that only F8 formulation is released $50 \%$ up to the 12 hours, so F8 is the optimized batch. As none of the batch has highest drug release so from the studies we can conclude that increase in the polymer amount and crosslinking agent amount shows negative effect with the drug release. ${ }^{[16]}$ The Table 4 is the cumulative drug release of all the formulations.

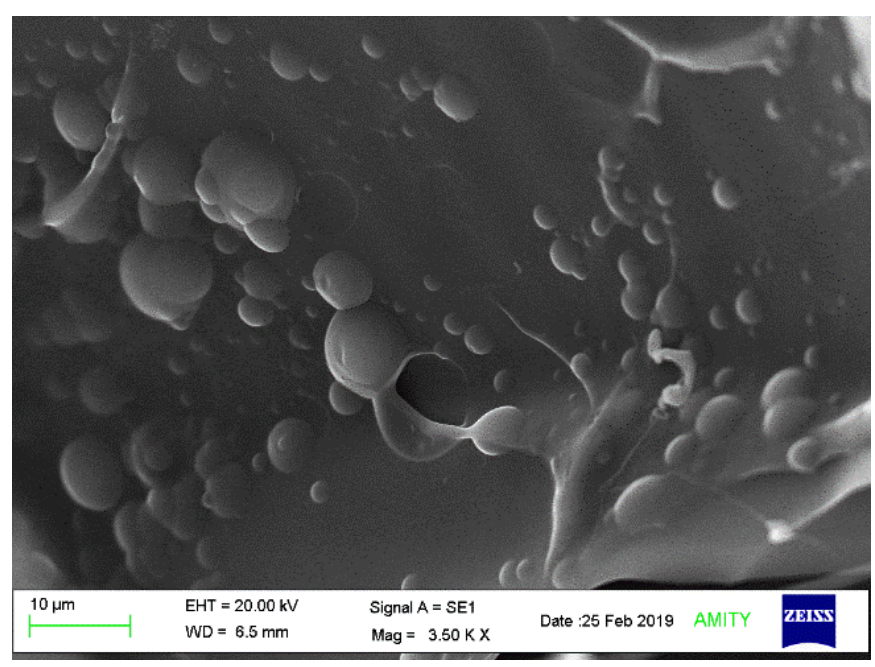

Fig. 3: SEM images of microparticles

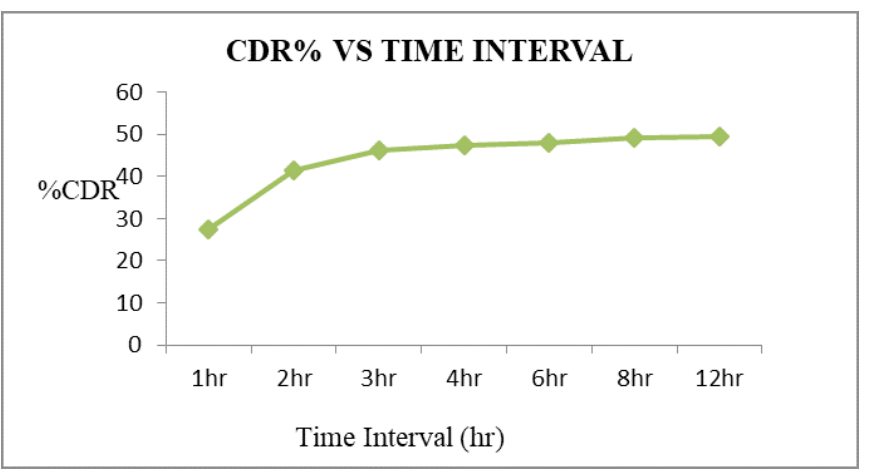

Fig. 4: In vitro release of optimized formulation
In-vitro Release Kinetics

The release kinetics of the formulation, which was optimized, was checked by fitting the results of the released data in the kinetic models. The release kinetic followed by the model is best fitted to the korsmeyer peppas model and it is shown in Fig. 5. ${ }^{[17]}$

\section{In-vitro Microbial Studies}

The MIC of the microparticles was checked against the bacteria strains. Antibacterial activity of microparticles was checked against Staphyllococcus aureus, Streptococcus mutans, Klebsiella pneumonia, and Escherichia coli. Prepared ceftriaxone loaded chitosan microparticles successfully decrease the MIC ${ }^{[18-19]}$ of the bacteria and hence have the potential to decrease associated toxicity. Table 5 shows the MIC of microparticle (Fig. 6).

The results of MIC for the antibacterial assay are in the range, and the bacteria Escherichia coli showed the best activity. The zone of inhibition of microparticle shows that the maximum zone was occupied by Escherichia coli giving the best activity.

Table 3: Entrapment efficiency of ceftriaxone loaded microparticles

\begin{tabular}{lll}
\hline S. No. & Formulations & Entrapment efficiency \pm S.D. \\
\hline 1 & F1 & $38.33 \% \pm 0.84$ \\
2 & F2 & $47.1 \% \pm 0.65$ \\
3 & F3 & $40.5 \% \pm 1.6$ \\
4 & F4 & $32.5 \% \pm 0.9$ \\
5 & F5 & $41.8 \% \pm 1.4$ \\
6 & F6 & $40.9 \% \pm 1.2$ \\
7 & F7 & $49.28 \% \pm 0.46$ \\
8 & F8 & $61.7 \% \pm 0.32$ \\
9 & F9 & $32.8 \% \pm 0.55$ \\
10 & F10 & $41.1 \% \pm 0.57$ \\
11 & F11 & $43.2 \% \pm 1.2$ \\
12 & F12 & $37.7 \% \pm 1.25$ \\
13 & F13 & $46.4 \% \pm 0.96$ \\
\hline
\end{tabular}

Table 4: \% cumulative drug release of all formulation

\begin{tabular}{|c|c|c|c|c|c|c|c|c|}
\hline \multirow[b]{2}{*}{ S. No. } & \multirow[b]{2}{*}{ Formulation } & \multicolumn{7}{|c|}{ \% Cumulative amount of drug release with respect to time. } \\
\hline & & 1 hour & 2 hours & 3 hours & 4 hours & 6 hours & 8 hours & 12 hours \\
\hline 1 & F1 & $17.9 \pm 0.35$ & $18.4 \pm 0.23$ & $18.5 \pm 0.43$ & $19.8 \pm 0.34$ & $20.3 \pm 0.22$ & $25.1 \pm 0.21$ & $32.4 \pm 0.63$ \\
\hline 2 & F2 & $13.0 \pm 0.49$ & $16.5 \pm 0.67$ & $19.7 \pm 0.96$ & $20.9 \pm 0.54$ & $23.6 \pm 0.46$ & $29.7 \pm 0.43$ & $35.1 \pm 0.45$ \\
\hline 3 & F3 & $11.0 \pm 0.23$ & $22.3 \pm 0.35$ & $23.0 \pm 0.15$ & $23.5 \pm 0.21$ & $24.4 \pm 0.64$ & $27.7 \pm 0 / 95$ & $31.2 \pm 0.76$ \\
\hline 4 & $\mathrm{~F} 4$ & $9.33 \pm 0.16$ & $10.6 \pm 0.45$ & $11.0 \pm 0.26$ & $16.1 \pm 0.34$ & $17.3 \pm 0.35$ & $23.1 \pm 0.67$ & $29.2 \pm 0.54$ \\
\hline 5 & F5 & $11.6 \pm 0.13$ & $13.3 \pm 0.91$ & $13.5 \pm 0.37$ & $15.2 \pm 0.27$ & $16.7 \pm 0.98$ & $20.7 \pm 0.23$ & $29.8 \pm 0.69$ \\
\hline 6 & F6 & $17.7 \pm 0.45$ & $19.2 \pm 0.28$ & $21.0 \pm 0.64$ & $24.8 \pm 0.26$ & $27.0 \pm 0.26$ & $31.5 \pm 0.56$ & $36.2 \pm 0.39$ \\
\hline 7 & F7 & $13.0 \pm 0.89$ & $14.1 \pm 0.83$ & $15.6 \pm 0.48$ & $18.9 \pm 0.43$ & $22.3 \pm 1.23$ & $30.3 \pm 0.46$ & $38.3 \pm 0.74$ \\
\hline 8 & F8 & $27.5 \pm 1.02$ & $41.4 \pm 0.74$ & $46.1 \pm 0.26$ & $47.9 \pm 0.29$ & $48.0 \pm 0.21$ & $49.3 \pm 0.26$ & $50.5 \pm 0.32$ \\
\hline 9 & F9 & $9.6 \pm 0.96$ & $11.0 \pm 0.15$ & $13.1 \pm 0.18$ & $15.2 \pm 0.65$ & $16.6 \pm 0.24$ & $20.2 \pm 0.57$ & $27.5 \pm 0.65$ \\
\hline 10 & F10 & $10.5 \pm 1.2$ & $13.1 \pm 0.97$ & $16.3 \pm 0.16$ & $17.0 \pm 0.49$ & $20.6 \pm 0.46$ & $26.1 \pm 0.94$ & $33.5 \pm 0.54$ \\
\hline 11 & F11 & $13.8 \pm 0.23$ & $14.9 \pm 0.91$ & $15.1 \pm 0.42$ & $17.6 \pm 0.78$ & $23.9 \pm 0.49$ & $29.7 \pm 1.05$ & $35.1 \pm 0.76$ \\
\hline 12 & F12 & $11.0 \pm 2.2$ & $12.7 \pm 1.06$ & $13.1 \pm 0.65$ & $15.6 \pm 0.65$ & $19.5 \pm 0.29$ & $25.3 \pm 2.01$ & $29.3 \pm 0.95$ \\
\hline 13 & F13 & $15.0 \pm 0.4$ & $17.8 \pm 1.09$ & $17.5 \pm 0.87$ & $19.6 \pm 0.32$ & $21.9 \pm 1.24$ & $23.6 \pm 1.34$ & $29.4 \pm 0.88$ \\
\hline
\end{tabular}


Table 5: Minimum inhibitory concentration of microparticles

\begin{tabular}{lll}
\hline & \multicolumn{1}{l}{ MIC in $\mu \mathrm{g} / \mathrm{mL}$} & \\
\cline { 2 - 3 } & $\begin{array}{l}\text { Formulation } \\
\text { (Ceftriaxone } \\
\text { microparticles) }\end{array}$ & $\begin{array}{l}\text { Control } \\
\text { (Ceftriaxone } \\
\text { marketed } \\
\text { formulation) }\end{array}$ \\
\hline Bacteria & $31.25-62.5$ & $0.78-1.56$ \\
Staphylococcus aureus & 15.6 & 0.78 \\
Klebsiela pneumoniae & 3.9 & $0.19-0.39$ \\
Escherichia coli & $0.95-1.9$ & $0.19-0.39$ \\
\hline
\end{tabular}

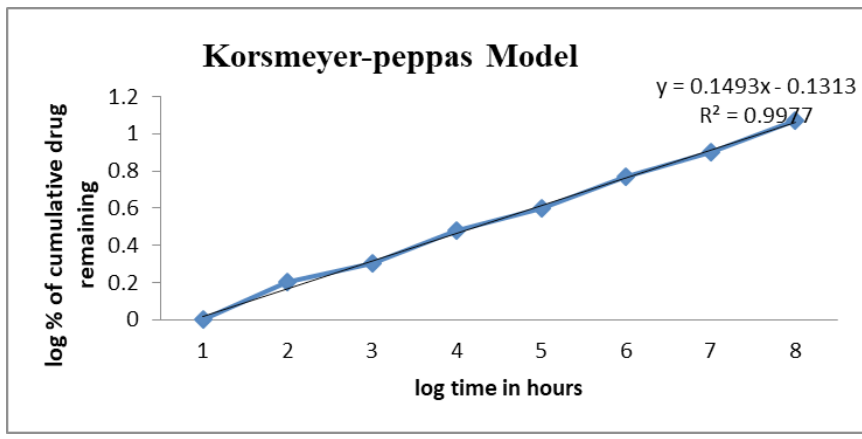

Fig. 5: In vitro release kinetics model

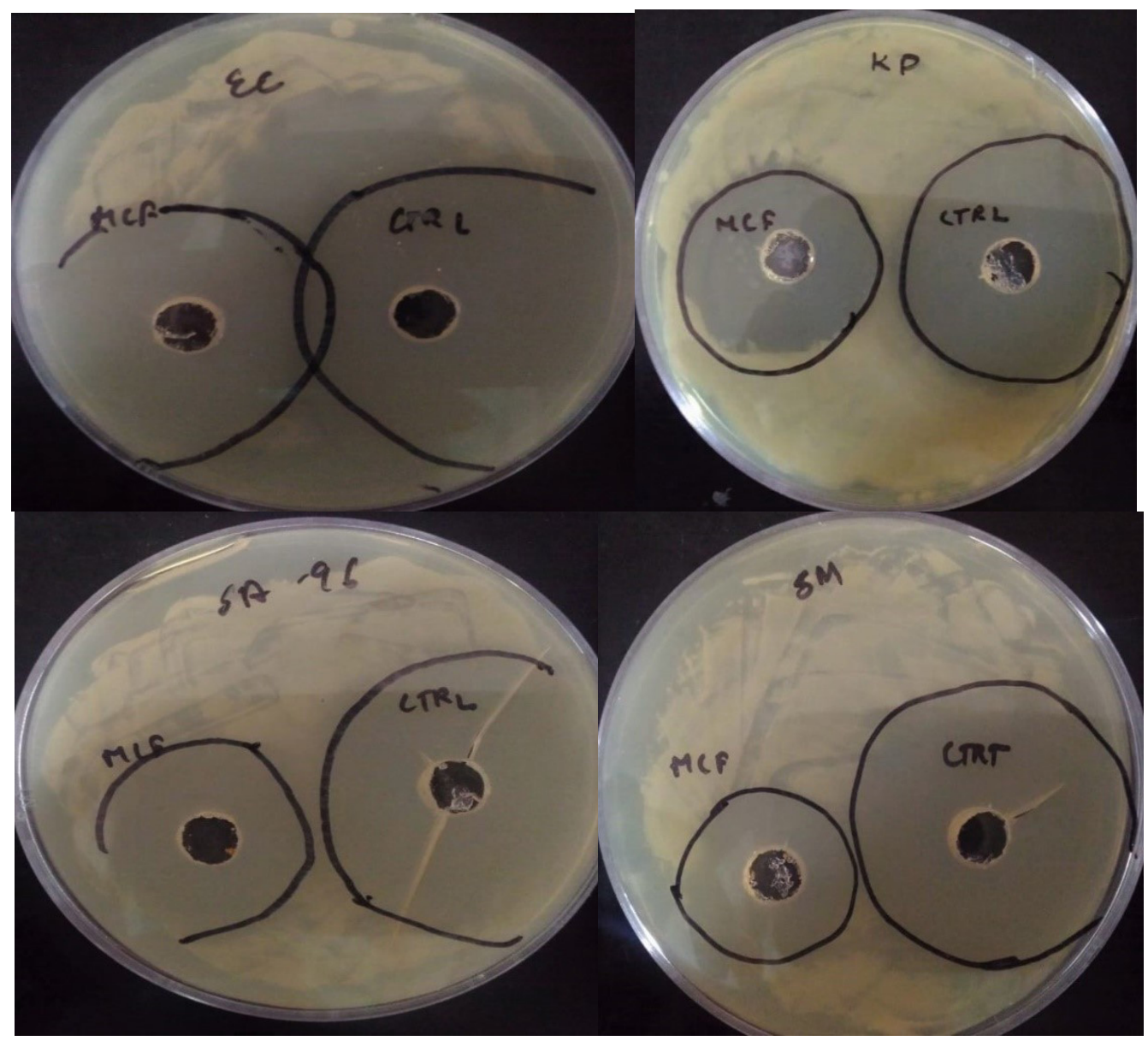

Fig. 6: Zone of inhibition of Escherichia coli , Klebsiella Pneumonia, Staphyllococcus Aureus, and Streptococcus Mutans

\section{CONCLUSION}

The aim of the studies is to formulate the chitosan-coated ceftriaxone loaded microparticulate drug delivery system and also to reduce the resistance occurring by checking the MIC in the different bacterial strains. The particle size of ceftriaxone microparticle batch eight was checked via DLS. Morphology of microparticle surface was determined using scanning electron microscopy and the optimized batch showed the smooth surface while checking it to the different proportion the size range of microparticle formed. ${ }^{[20]}$ In-vitro release showed only $50 \%$ of drug release at 12 hour of the final optimized formulation and entrapment efficiency was also highest in F8 formulation $61 \%$, which showed that there is a positive effect of drug polymer ratio and crosslinking agent with the increase in both of the parameter there is an increase in the encapsulation efficiency. The above result of the release of drug was the best fit in the korsmeyer peppas model, which showed that it is non fickian diffusion. The above results indicate that the ceftriaxone microparticles conclusion could be formulated to release the drug in the vicinity of microorganisms which could aid in decreasing the MIC of antibiotics and hence reduce toxicity. Prepared ceftriaxone 
loaded chitosan microparticles successfully decrease the MIC of the bacteria and hence have the potential to decrease associated toxicity.

\section{ACKNOWLEDGMENT}

The researchers would like to thanks the Amity Institute of Pharmacy for the financial supports of this study.

\section{REFERENCE}

1. Miller EL. The penicillins: a review and update. J midwifery \& women's health. 2002;47(6):426-434. Available from: https://www. ncbi.nlm.nih.gov/pubmed/12484664

2. Metkari VB, Kulkarni LV, Patil PS, Jadhav PA, Bamane GS, Kumbhar CM. Microspheres-a new drug delivery system: A review. Current Pharma Research. 2014;4(2):1128-1133.

3. Midha K, Nagpal M, Arora S. Research article: Microsphere a recent update. 2015;6(8):5859-5867.

4. Kumbar SG, Kulkarni AR, Aminabhavi TM. Crosslinked chitosan microspheres for encapsulation of diclofenac sodium: Effect of crosslinking agent. J Microencapsulation. 2002; 19(2): 173-180.

5. Thanoo BC, Sunny MC, Jayakrishnan A. Cross-linked Chitosan Microspheres: Preparation and Evaluation as a Matrix for the Controlled Release of Pharmaceuticals. J Pharm Pharmacol. 1992; 44(4):283-286.

6. Kataria S, Sahil K, Akanksha M, Premjeet S, Bilandi A, Kapoor B. Microsphere: a Review. Int J Res Pharm Chem [Internet]. 2011;1(4): 1184-1198. Available from: https://pdfs.semanticscholar.org/f59 2/7344cb08b61ee78a43cf2f11ac0c4fd00734.pdf

7. Ghadi A, Mahjoub S, Tabandeh F. Synthesis and optimization of chitosan nanoparticles: Potential applications in nanomedicine and biomedical engineering. Casp J Intern Med. 2014;5(3):156161.

8. Murueva AV, Shershneva AM, Abanina KV., Prudnikova SV., Shishatskaya EI. Development and characterization of ceftriaxoneloaded P3HB-based microparticles for drug delivery. Dry Technol
[Internet]. 2019;37(9): 1131-1142. Available from: https://doi.org /10.1080/07373937.2018.1487451

9. Patel K, Patel M. Preparation and evaluation of chitosan microspheres containing nicorandil. Int J Pharm Investig. 2014;4(1):32.

10. Rashid M, Arabia S, Yusuf M, Arabia S, Khan M, Arabia S. Cumin and Residronate loaded chitosan microparticles for treatment of osteoporosis. Int J Pharma Sci Res. 2018;9(07):83-98.

11. Santeesh Mahav, N V \& Kala S. Crosslinked chitosan microspheres for encapsulation of diclofenac sodium: effect of crosslinking agent. Int J PharmTech Res [Internet]. 2011;3:1242-1254.

12. Wong LP. Preparation and characterisation of sustained-release. Int J Pharm. 1992;83(8):955-114.

13. Pathak A, Shrivasatava N, Baghel S. A review on microspheres: methods of preparation and evaluation. World J Pharm Pharm Sci. 2012;1(1):422-438.

14. Ruangpan L. Chapter 3. Minimal inhibitory concentration (MIC) test and determination of antimicrobial resistant bacteria. Lab Man Stand methods Antimicrob Sensit tests Bact Isol from Aquat Anim Environ [Internet]. 2004;(Mic):31-55. Available from: http://hdl. handle.net/10862/1637

15. Tiruwa R. A review on nanoparticles - preparation and evaluation parameters. Indian J Pharm Biol Res. 2015;4(2):27-31.

16. Kauss T, Marchivie M, Phoeung T, Gaubert A, Désiré A, Tonelli G, et al. Preformulation studies of ceftriaxone for pediatric non-parenteral administration as an alternative to existing injectable formulations. Eur J Pharm Sci [Internet]. 2017;104(April):382-392. Available from: http://dx.doi.org/10.1016/j.ejps.2017.04.010

17. Bansal H, Preet kaur S, Gupta AK. Microsphere: Methods of prepration and applications; A comparative study. Int J Pharm Sci Rev Res. 2011;10(1):69-78.

18. Abdellatif AA. Microparticles Formulation as a Targeting Drug Delivery System. J Nanomedicine Res. 2017;6(2):0-4.

19. Thakkar H, Murthy R. Effect of cross-linking agent on the characteristics of celecoxib loaded chitosan microspheres. Asian J Pharm. 2008;2(4):246.

20. Anilkumar J. Shinde and Harinath N., Formulation, development and characterization of Simvastatin nanoparticles by solvent displacement method, Der Pharmacia Lettre, 2014;6:2:145155. 